Running head: ERROR-MONITORING IN MAJOR DEPRESSION

Electrical brain imaging reveals the expression and timing of altered error monitoring functions in major depression

Kristien Aarts ${ }^{1}$, Marie-Anne Vanderhasselt ${ }^{1}$, Georges Otte ${ }^{2}$, Chris Baeken ${ }^{3}$, and Gilles Pourtois ${ }^{1}$

${ }^{1}$ Department of Experimental Clinical and Health Psychology, Ghent University, Belgium

${ }^{2}$ P.C. Dr. Guislain, Fr. Ferrerlaan 88A, 9000 Ghent - Belgium

${ }^{3}$ Department of Psychiatry, University Hospital, Ghent University, Belgium

Correspondence:

Kristien Aarts

Department of Experimental Clinical and Health Psychology

Ghent University

Henri Dunantlaan 2

9000 Ghent

Belgium

Phone: +3292649413

Email: kristien_aarts@hotmail.com 


\begin{abstract}
Major depressive disorder (MDD) is characterized by disturbances in affect, motivation, and cognitive control processes, including error detection. However, the expression and timing of the impairments during error monitoring remain unclear in MDD. Accordingly, the behavior and event-related brain responses (ERPs) of 20 patients with MDD were compared to those of 20 healthy controls (HCs), while they performed a Go/noGo task. Errors during this task were associated with two ERP components, the error-related negativity (ERN/Ne) and the error positivity (Pe). Results show that the ERN/Ne-correct-related negativity (CRN) amplitude difference was significantly larger in MDD patients (after controlling for speed), compared to HCs, while MDD patients exhibited overactive medial frontal cortex (MFC) activation. By comparison, the subsequent Pe component was smaller in MDD patients compared to HCs and this effect was accompanied by a reduced activation of ventral anterior cingulate cortex (ACC) regions. These results suggest that MDD has multiple cascade effects on early error monitoring brain mechanisms.
\end{abstract}

Keywords: Major depression, action-monitoring, error-related negativity (ERN), error positivity $(\mathrm{Pe})$ 


\section{Electrical brain imaging reveals the expression and timing of error monitoring functions in major depression}

A prefrontal-limbic dysregulation seems to be related to the onset and maintenance of MDD (Mayberg, 1997) and this prefrontal-limbic network has also been associated with the detection of response errors (Bush, Luu, \& Posner, 2000; Pourtois et al., 2010; Seifert, von Cramon, Imperati, Tittgemeyer, \& Ullsperger, 2011). In light of this evidence, errormonitoring functions should thus be deficient in MDD. Indeed, Holmes and Pizzagalli (2008) showed that depression is associated with an increased activation within midline prefrontal regions. They observed increased activity in the rostral Anterior Cingulate Cortex (rACC) and the medial Prefrontal Cortex (PFC) 80 ms after error commission, as well as a disrupted connectivity between the rACC and the left dorsolateral PFC. While in healthy controls (HCs), increased ACC activity predicted the activity in the left dlPFC $\sim 472 \mathrm{~ms}$ after error commission. A similar relationship was not found for MDD patients.

Event related potential (ERP) experiments looking at error-monitoring in MDD have focused on two components, the error-related negativity (ERN/Ne), and the error positivity (Pe) (Falkenstein, Hoormann, Christ, \& Hohnsbein, 2000). The ERN/Ne is a negative deflection peaking $~ 0-50 \mathrm{~ms}$ following an incorrect response with a maximum amplitude over fronto-central midline sites (Falkenstein, Hohnsbein, Hoormann, \& Blanke, 1991; Gehring, Goss, Coles, Meyer, \& Donchin, 1993). The ERN/Ne component is followed by a large positivity, the Pe. This component reaches its maximum amplitude over centro-parietal scalp recordings along the midline $\sim 200-400 \mathrm{~ms}$ post-error onset (Falkenstein et al., 1991; Overbeek, Nieuwenhuis, \& Ridderinkhof, 2005). Unlike the ERN/Ne, the Pe is thought to reflect a conscious stage of error detection (Nieuwenhuis, Ridderinkhof, Blow, Band, \& Kok, 2001). Alternatively, it could reflect an affective appraisal of errors (Falkenstein et al., 2000), 
a P300-like orienting response (Ridderinkhof, Ramautar, \& Wijnen, 2009), or the accumulation of evidence that an error has been committed (Steinhauser \& Yeung, 2010).

Each of the two error-related ERP components was shown to vary with MDD. However, mixed results were obtained regarding the nature and direction of these MDDrelated changes. While some studies found a larger ERN/Ne in MDD patients compared to HCs (Chiu \& Deldin, 2007; Holmes \& Pizzagalli, 2008; Holmes \& Pizzagalli, 2010), other studies reported similar (Schrijvers, de Bruijn, et al., 2008; Schrijvers et al., 2009) or smaller ERN/Ne amplitudes in MDD patients (Ruchsow et al., 2006; Ruchsow et al., 2004). Likewise, discrepant findings have been reported regarding amplitude variation of the $\mathrm{Pe}$ component. While Chiu and Deldin (2007), and Holmes and Pizzagalli (2008) reported similar Pe amplitudes for HCs and MDD patients, Schrijvers, de Bruijn, et al. (2008) and Schrijvers et al. (2009) reported smaller Pe amplitudes in MDD patients compared to HCs.

An explanation for these discrepant findings may be that the amplitude of the ERP signal might not be different between MDD and HCs when measured at a few electrode positions, but rather that the expression of the electric field would be different in depression, consistent with a change in the underlying neural generators. However, these topographical changes are usually difficult to capture using standard peak measurements (Pourtois, Delplanque, Michel, \& Vuilleumier, 2008). Hence, the question arises whether when using alternative data analyses, we could find evidence for a change in prefrontal and anterior cingulate brain areas giving rise to the ERN/Ne and Pe components as a function of MDD.

The goal of this ERP study was to better characterize possible changes in early error monitoring brain processes (with a focus on the ERN/Ne and Pe components) in MDD patients. Using 128-channel EEG, the electrophysiological responses to commission errors performed during a Go/noGo task were compared between MDD patients and HCs. Because previous ERP studies reported mixed results regarding amplitude modulations of the ERN/Ne 
component as a function of depression (Chiu \& Deldin, 2007; Compton et al., 2008; Holmes \& Pizzagalli, 2008; Holmes \& Pizzagalli, 2010; Schrijvers, . 2008; Schrijvers et al., 2009), we did not formulate a clear directional prediction regarding a possible change of the amplitude of the ERN/Ne in MDD. However, given that MDD is typically conceived as an internalizing disorder (Mineka, Watson, \& Clark, 1998) and because the ERN/Ne is thought to be reliably enhanced or overactive in this type of disorders, we surmised that MDD patients might show a larger ERN/Ne than the HCs (Olvet \& Hajcak, 2008). Moreover, it was predicted that this effect might be associated with altered activities in MFC regions, including the dACC (see also Holmes \& Pizzagalli, 2008). Regarding effects of MDD on the subsequent Pe component, no hypothesis was formulated since previous ERP studies reported mixed results for amplitude variations of this mid-latency error-related activity as a function of depression (Chiu \& Deldin, 2007; Holmes \& Pizzagalli, 2008; Holmes \& Pizzagalli, 2010; Schrijvers, de Bruijn et al., 2008; Schrijvers et al., 2009).

\section{Methods}

\section{Participants}

Twenty-three non-depressed HCs (18 females; mean age: $39, S E M=3.04)$ and 25 individuals meeting the Diagnostic and Statistical Manual of Mental Disorders criteria (DSM-IV-TR; American Psychiatric Association) for MDD (15 females; mean age: 38, SEM $=2.55)$ participated in this study. The data of 8 participants had to be excluded because they did not commit enough errors (i.e., $<6$; 2 HCs and 5 MDD patients) or the raw EEG data were contaminated by many artifacts (i.e., more than $20 \%$ precluding the possibility to compute reliable ERP waveforms; $1 \mathrm{HC}$ ). The demographic and clinical data of these 8 participants were comparable to the ones of the participants eventually included in the study, all $p$-values $>.10$. In total, the data of $20 \mathrm{HCs}(17$ females; mean age: $39, S E M=3.43)$ and 
20 MDD patients (10 females; mean age: $37, S E M=2.85)$ were included in the analyses. Demographic and clinical characteristics are outlined in Table 1.

The MDD outpatients were recruited from a psychiatric clinic. All patients were selected by a psychiatrist using the Mini-International Neuropsychiatric Interview (MINI; Sheehan et al., 1998), a structured clinical interview, and they were all diagnosed with unipolar major depression of the melancholic subtype (ICD-9-CM code 296.23 and 296.33) according to the DSM-IV-TR (American Psychiatric association, 2000). Severity of depression was assessed with the 17-item Hamilton Depression Rating Scale (HDRS; Hamilton, 1967) and the 21item Beck Depression Inventory (BDI-II; Beck \& Steer, 1984). A psychiatrist rated depression symptoms and severity (HDRS). Moreover, the Hamilton Rating Scale for Depression (HAM-D; Hamilton, 1960) and the MINI, were administered again one week before testing to examine the severity of the current MDD episode (HAM-D: $M=28.65$; $S E M=1.17$; see Table 1). Finally, levels of depression were again verified at testing, using the Beck Depression Inventory (BDI-II; Beck, Steer, \& Brown, 1996) and the HAM-D (Hamilton, 1967). These scores confirmed that all patients who were previously diagnosed as clinically depressed, were still found to be clinically depressed at the day of testing (see Table 1). Exclusion criteria were 1) other mood disorders than MDD (comorbid anxiety disorders were allowed; specific phobia: $n=1$; post-traumatic stress disorder: $n=1$; social anxiety: $n=1$ ); 2) the use of anti-psychotics, tricyclic anti-depressants and/or long lasting benzodiazepines; 3) a history of neurological disorder, including epilepsy, head injury, and loss of consciousness; 4) a history of electroconvulsive therapy; 5) alcohol abuse during the past year; 6) a past or present substance dependence; 7) past or present experience of psychotic episodes; and 8) learning disorders. During the test session, all MDD participants were on either Selective Serotonin Reuptake Inhibitors (SSRI) or Selective Noradrenalin Reuptake Inhibitors (SNRI). Nine out of 20 patients were taking anti-depressant medication for a 
duration of at least 7 months before testing and could therefore be considered as being treatment-resistant. However, despite this prolonged pharmacological treatment, they still met the criteria for MDD. HCs were recruited using advertisements in newspapers and were free of any medication. By contrast, HCs reported that they had never been diagnosed with MDD or another psychiatric disorder prior to the EEG testing. This was also verified by the MINI that was administered at testing.

All participants were Dutch speakers, gave their written informed consent, and received a compensation of 20 Euros. The study was approved by the medical ethics committee of the Ghent University hospital.

\section{Stimuli and task}

Participants performed a speeded Go/noGo task that was previously used and validated (Figure 1; Vocat et al., 2008) . Visual stimuli were shown on a 17-inch LCD screen. They consisted of an arrow $\left(11.4^{\circ} \times 0.05^{\circ}\right.$ visual angle at a $60 \mathrm{~cm}$ viewing distance $)$ that was presented in the center of the screen on a white background. Each trial started with a fixation cross that lasted for 1000 ms. Then, a black arrow, oriented either up or down, was presented. After a variable interval (1000-2000 ms) the black arrow became either green or turquoise while its orientation could either remain identical or shift in the opposite direction. Participants were asked to perform a speeded color plus orientation discrimination task. When the black arrow turned green and the orientation remained unchanged $\left(2 / 3^{\text {rds }}\right.$ of the trials), participants were instructed to press a predefined key on the response box as fast as possible with the index finger of their dominant hand (Go trials). However, participants had to withhold responding when either the arrow became green but changed orientation $\left(1 / 6^{\text {th }}\right.$ of the trials), or when the arrow became turquoise and kept its initial orientation $\left(1 / 6^{\text {th }}\right.$ of the trials), enabling two types of noGo trials. For noGo trials, this color arrow remained on the 
screen for a maximum duration of $1000 \mathrm{~ms}$. Instructions emphasized both speed and accuracy.

Given that the ERN/Ne amplitude varies according to the number of errors (i.e., the ERN/Ne is larger when response errors are less frequent; see Gehring et al, 1993), it was important to avoid obvious group differences regarding error rate. Therefore, to ensure that the number of response errors was balanced between MDD patients and HCs, a specific procedure was used topromote the occurrence of fast RTs, and accordingly the commission of errors on noGo trials.

The experiment consisted of a practice block of 12 trials (4 Go, 4 noGo of each condition), 3 calibration blocks of 14 trials (10 Go and 2 noGo of each type) and 6 test blocks of 60 trials ( 40 Go trials and 20 noGo trials). Each calibration block was followed by two test blocks. Trial presentation was randomized within blocks. Stimulus presentation and response recording were controlled using E-prime software (V2.0., http://www.pstnet.com/products/eprime/).

\section{Analysis of behavioral data}

RTs faster than $150 \mathrm{~ms}$ (Error: $M=.79, S E M=.33$; Hit: $M=.33, S E M=.15$ ) and slower than $800 \mathrm{~ms}$ (Error: $M=2.25, S E M=.91$; Hit: $M=1.47, S E M=.36$ ) were removed from the analyses. Next, RTs faster than $M-2.5 S D$ (Error: $M=.14, S E M=.14$; Hit: $M=$ $.01, S E M=.01$ ) or slower than $M+2.5 S D$ (Error: $M=2.25, S E M=.43$; Hit: $M=2.76, S E M$ $=.16)$ were also excluded. The number of outliers was not significantly different between HCs and MDD patients, all $p>.10$, except for RTs for Hits. MDDs reacted slower than 800 ms $(M=2.17, S E M=.65)$ more often than HCs $(M=.77, S E M=.24)$ in the Hit condition. Color and orientation errors were collapsed together (error condition) since there was no significant group difference regarding accuracy between these two error types, $t<1$. However, a significant difference was observed in the number and RT speed between color 
and orientation errors (accuracy: color errors: $M=10 ; S E M=1.19$; orientation errors: $M=$ $15 ; S E M=1.57 ; t(39)=-5.95, p<.001)(\mathrm{RT}$ speed: color errors: $M=258 \mathrm{~ms} ; S E M=8.45$; RT orientation errors: $M=306 ; S E M=11.94 ; t(37)=-4.95, p<.001)$. This result indicated a propensity to commit more false alarms with orientation changes than color changes in this task. However, this effect was comparable for MDD patients and HCs, $F<1$. Fast and slow hits were also collapsed and treated as a single condition (hit condition). Mean RTs for errors and hits as well as the number of errors and hits were then computed and compared by means of $2 \times 2$ mixed analyses of variance (ANOVAs), with group (HC vs. MDD) as betweensubjects factor and accuracy (Error vs. Hit) as within-subject variable. Finally, the classical post-error slowing and post-error accuracy effects (Laming, 1979; Rabbitt, 1966) were calculated to ascertain that in both groups errors were processed similarly as distinctive events, compared to hits.

\section{EEG recording}

EEG was acquired at $512 \mathrm{~Hz}$ using a 128-channel Biosemi Active Two system (http://www.biosemi.com) referenced to the Common Mode Sense (CMS) active electrode Driven Right Leg (DRL) passive electrode. ERPs of interest were computed offline following a standard sequence of data transformations (Picton et al., 2000): (1) -500/+1000 segmentation around the onset of the response, (2) pre-response interval baseline adjustment (from -500 ms to response onset), (3) vertical ocular correction for blinks (Gratton, Coles, \& Donchin, 1983), using the difference amplitude of two electrodes attached above and below the left eye (Note that no correction for horizontal eye movements was performed using this procedure. Artifacts related to these horizontal eye movements were removed manually during the artifact rejection step), (4) artifact rejection $[M=-87.25 /+87.25, S E M=2.24$ amplitude scale $(\mu \mathrm{V})$ across participants; no significant difference between HCs $(M=89.00$, $S E M=2.98)$ and MDD patients $(M=85.50, S E M=3.36), t<1],(5)$ averaging of trials, 
separately for each group (HC vs. MDD) and experimental condition (errors vs. hits), and (6) $30 \mathrm{~Hz}$ low pass digital filtering of the individual average data.

\section{Standard peak analyses}

For each of the two error-related ERP deflections and for each condition, the area under the curve was calculated and analyzed (Picton et al., 2000). This was done during the 25-55 ms interval post response onset at electrode $\mathrm{FCz}$ for the ERN/Ne amplitude, and during the 150-210 ms interval post-response onset at electrode $\mathrm{Cz}$ for the Pe component. The selection of these two specific scalp locations (and time windows) was based on the topographic properties of the present dataset.

Statistical analyses were performed on the mean amplitude of each area using a 2 (accuracy: error vs. hit) x 2 (group) repeated measures ANOVA, with the alpha cutoff set to $p$ $<.05$.

\section{Topographical analyses}

A complementing topographic mapping analysis of the ERP data was performed (see Figure 2Pourtois et al., 2008) . This pattern analysis summarizes ERP data into a smaller number of dominant field configurations, previously referred to as functional microstates (Lehmann \& Skrandies, 1980; Michel, Seeck, \& Landis, 1999). The rationale and basic principles of this temporal segmentation method have already been extensively described elsewhere (Michel et al., 1999; Murray, Brunet, \& Michel, 2008; Pourtois et al., 2008). Following standard practice, a topographic pattern analysis was first performed on the grandaverage ERP data from $-55 \mathrm{~ms}$ until $379 \mathrm{~ms}$ after response onset (222 consecutive time frames at $512 \mathrm{~Hz}$ sampling rate, encompassing the ERN/Ne and Pe components) using a standard K-means cluster method (Pascual-Marqui et al., 1995). The dominant scalp topographies (identified by the previous analysis) were then fitted back to the ERP data of each subject using spatial fitting procedures to quantitatively determine their representation 
across subjects and conditions. GEV represents the sum of the explained variance weighted by the Global Field Power (GFP) at each moment in time. The resulting GEV values were entered in ANOVAs with two within-subject factors: accuracy (errors vs. hits) and map configuration (i.e., the dominant electric field distributions identified by the spatial cluster analysis), as well as group (HC vs. MDD) as the between-subjects factor. These analyses were carried out using CARTOOL software (Version 3.34; developed by D. Brunet, Functional Brain Mapping Laboratory, Geneva, Switzerland).

\section{Source localization analyses}

Finally, to estimate the neural generators underlying the dominant error-related electrical field configurations identified by the previous analyses, a distributed linear inverse solution was used, namely standardized low-resolution brain electromagnetic tomography (sLORETA, Pascual-Marqui, 2002). SLORETA solutions are computed within a three-shell spherical head model co-registered to the MNI152 template (Mazziotta et al., 2001). SLORETA estimates the 3-dimensional intracerebral current density distribution in 6239 voxels (5 $\mathrm{mm}$ resolution), each voxel containing an equivalent current dipole. This 3dimensional solution space in which the inverse problem is solved, is restricted to the cortical gray matter. The head model for the inverse solution uses the electric potential lead field computed with a boundary element method applied to the MNI152 template (Fuchs, Kastner, Wagner, Hawes, \& Ebersole, 2002). Scalp electrode coordinates on the MNI brain are derived from the international 5\% system (Jurcak, Tsuzuki, \& Dan, 2007). A direct comparison between the inverse solution results of MDD patients and HCs was performed separately for the ERN/Ne and Pe component, using unpaired $t$-tests. To reveal group effects at the statistical level using a corrected $\mathrm{p}<.05$ value, a stringent non-parametric randomization test (relying on 5000 iterations) was used. The calculation of all reconstruction parameters 
was based on the computed common average reference. SLORETA units were scaled to ampere per square meter $\left(\mathrm{A} / \mathrm{m}^{2}\right)$.

\section{Results}

\section{Behavior}

Accuracy (errors vs. hits) and RT data are presented in Table 2. The number of errors was similar between MDD patients and $\mathrm{HCs}, t<1$. All participants were faster for errors than for hits, $F(1,38)=43.21, p<.001$, but overall, MDD patients reacted slower than HCs, $F(1$, $38)=5.53, p<.05$. Importantly, this latter speed effect did not interact with accuracy (error vs. hit), $F(1,38)=1.13, p>.10$. A classical post-error slowing effect (Laming, 1979; Rabbitt, 1966), indicated by slower RTs for Hits following errors compared to Hits following Hits, was evidenced, $F(1,38)=6.96, p<.05$. This adaptation effect did not interact with group, $F$ < 1. Moreover, no Laming effect (Laming, 1979) or difference between post-error vs. post-hit accuracy was noted, $F<1$, equally so in both groups, $F<1$.

\section{ERP components}

A clear negative deflection was observed $\sim 40 \mathrm{~ms}$ after error commission with maximum amplitude over fronto-central electrodes (e.g., FCz). These electrophysiological properties were compatible with the ERN/Ne (Figure $3 \mathrm{AB}$ ). This early negative component was larger following errors compared to hits, $F(1,38)=5.33, p<.05$. Although this difference appeared to be larger for MDD patients $(M=2.09 ; S E M=.80 ; t(19)=-2.61, p<$ $.05)$ than for HCs $(M=.70 ; S E M=.90 ; t<1)$, there was no significant effect of group, $F(1$, $38)=1.53, p>.10$, nor a significant interaction between accuracy (error vs. hit) and group $(\mathrm{HC}$ vs. $\mathrm{MDD}), F(1,38)=1.33, p>.10$ (Figure $3 \mathrm{CD})$. However, this interaction became significant when including speed as a covariate given that on average MDD patients were slower than $\mathrm{HCs}, F(1,37)=4.64, p<.05$. More specifically, post-hoc comparisons showed a difference between the ERN and CRN in the MDD group, $F(1,18)=10.83, p<.01$, while 
this difference only reached a trend towards significance in the $\mathrm{HC}$ group, $F(1,18)=3.79, p$ $=.07$. Consistent with previous ERP studies using this Go/noGo task (Aarts \& Pourtois, 2010; Dhar \& Pourtois, 2011), and given the speed pressure imposed on participants as well as the relatively high number of errors committed within a short period of time, the ERN/NeCRN amplitude difference was actually modest at this specific electrode position $(\mathrm{FCz})$, though being significant, suggesting that response errors were discriminated from hits early on following response onset, especially so for MDD patients.

The ERN/Ne was followed by a large positive component that reached its maximum amplitude at central electrodes along the midline (i.e., $\mathrm{Cz}$ ) and that was clearly modulated in size by accuracy (errors vs. hits). More specifically, this positive deflection was reliably larger for errors compared to hits, $F(1,38)=85.80, p<.001$. These properties (latency, polarity, topography) were compatible with the generation of a genuine Pe component during early error detection. This positive component was larger in HCs than in MDD patients, $F(1$, $38)=6.70, p<.05$, but this effect did not interact with accuracy, $F(1,38)=2.26, p>.10($ see Figure 3EF). An auxiliary analysis including speed as a covariate confirmed this statistical outcome (i.e., accuracy, $F(1,37)=4.61, p<.05$; group, $F(1,37)=4.50, p<.05$; accuracy $\mathrm{x}$ group: $F(1,37)=1.54, p>.10)$.

Furthermore, to assess if MDD had a differential impact on the ERN and Pe components, a 2 (accuracy: error vs. hit) x 2 (ERP component: ERN vs. Pe) x 2 (group: HCs vs. MDDs) ANOVA was carried out. This analysis showed significant effects of accuracy, $F(1,38)=$ $16.60, p<.001$, and of ERP component, $F(1,38)=201,38, p<.001$. Whereas the interaction term between ERP component, accuracy and group remained non-significant, $F>1$, a significant main effect of group was evidenced ${ }^{1}, F(1,38)=5.30, p<.05$. Hence, based on

\footnotetext{
${ }^{1}$ We also assessed whether the putative measurement error was similar for the ERN and Pe components and for the two groups. For this purpose, we compared the standard deviation (inter-individual variability) of the ERN
} 
this auxiliary analysis, we could conclude that MDD was not differently related to the Pe vs. ERN. Instead, the ERN was augmented in the MDD group, when controlling for RT differences across the two groups, and the Pe was smaller in MDD patients relative to HCs. Complementary topographical and source localization analyses were therefore used to assess if MDD, during each of these two consecutive moments, was differently related to the neural processing of these salient events in non-overlapping cortical brain areas compared to HCs.

\section{Topographical components}

A solution with 8 dominant maps explained $94 \%$ of the variance. Next, an analysis was performed on the dominant maps generated during the time interval corresponding to the ERN/Ne and Pe, and their likely variations as a function of accuracy and/or group.

During the time interval corresponding the ERN/Ne vs. CRN component (starting 10 ms - before response onset and ending 90 ms post-response onset), a main change in the topography between errors and hits was evidenced. Whereas the topography for hits was characterized by a broad negative activity extending towards prefrontal sites (CRN map), the scalp distribution for response errors was qualified by a negative activity circumscribed to a few precentral electrode positions, including $\mathrm{FCz}$ (Figure 4A; ERN/Ne map). This ERN topography showed a left lateralization, an observation which could potentially be explained by the mono-manual (i.e., right hand) stimulus-response mapping used with this Go/noGo task (Aarts \& Pourtois, 2010; Gruendler, Ullsperger, \& Huster, 2011). This result suggests that beyond local amplitude variations found for the peak of the ERN/Ne component as measured at electrode FCZ, errors are also associated with a change in the topography of the electric field compared to hits. This finding therefore suggested that the brain network giving

and Pe within each group as well as across the two groups. We also compared the standard deviation of the difference between the mean of the ERN and Pe (see Masson \& Loftus, 2003). These analyses showed comparable measurement error for these two ERP components and two groups. 
rise to response errors vs. hits could be dissociated. These two dominant maps were fitted back to the individual ERP data to verify whether this topography-related change during the ERN/CRN was significant (and different across the two groups) or not. The GEV values obtained for these two dominant maps after fitting were therefore submitted to a 2 (map) $\mathrm{x} 2$ (group) x 2 (accuracy) repeated measures ANOVA. This analysis revealed a significant interaction between accuracy and map/scalp configuration, $F(1,38)=44.04, p<.001$. While the CRN map explained more variance for hits than errors, $t(39)=-8.06, p<.001$, the ERN/Ne map had a symmetric profile, explaining more variance for errors than hits, $t(39)=$ 2.66, $p<.05$. However, this interaction effect was similar for MDD patients and HCs, $F<1$ (Figure 4B).

Regarding the time interval corresponding to the Pe component $(\sim 145-281 \mathrm{~ms}$ post response onset), a specific error-related topography (Pe map, with a maximum amplitude at electrode CZ) was identified. By contrast, hits elicited a distinct posterior positivity (see Figure 4C). Further analyses computed on the mean GEV values obtained for these two dominant maps confirmed a significant interaction between accuracy and map, $F(1,38)=$ 28.55, $p<.001$. Whereas the Pe map explained more variance for errors than hits, $t(39)=$ $5.39, p<.001$, the other concurrent map (posterior positivity map) showed a symmetric effect, explaining more variance for hits than errors, $t(39)=-4.21, p<.001$. Interestingly, this analysis also showed a significant interaction between map and group, $F(1,38)=7.17, p=$ .01 (Figure 4D). This interaction was explained by the fact that the Pe map explained more variance for errors committed by HCs than MDD patients, $t(38)=3.67, p<.001$. The same effect was evidenced, though much weaker, for hits, $t(38)=1.92, p=.06$. However, the concurrent posterior positivity map associated with hits was not significantly different between groups, both for errors, $t(38)=-1.37, p>.10$, and hits, $t<1$, suggesting that MDD was primarily associated to an altered neural processing of errors, but not hits. 


\section{Inverse solutions}

To gain insight into the configuration of the intracranial generators underlying the global topographic-dependent changes, the intracranial generators of the ERN/Ne and Pe maps were estimated using sLORETA (Pascual-Marqui, 2002).

This analysis confirmed that the configuration of the intracranial generators underlying the ERN/Ne scalp map (errors) were similar between HCs and MDD patients. These generators primarily involved MFC/dACC regions, consistent with several earlier studies (Debener, Ullsperger, Fiehler, von Cramon, \& Engel, 2005; Dehaene, Posner, \& Tucker, 1994; Herrmann, Rommler, Ehlis, Heidrich, \& Fallgatter, 2004; O'Connell et al., 2007). For $\mathrm{HCs}$, the neural generators of the ERN/Ne were mainly localized within superior frontal gyrus/dACC (maximum: 6x, 6y, 44z; Brodmann Areas (BAs) 32, 24 and 6). For MDD patients, they also involved the superior frontal gyrus/dACC (maximum: 6x, 6y, 44z; BAs 6, 8, 32 and 24), but with a slight shift towards the front for the maxima, compared to HCs (Figure 5A). Importantly, a statistical comparison in the inverse solution space (see Table 3) between the two groups showed that MDD patients had a significantly stronger MFC/dLPFC (BA6, BA8 and BA9) activation than HCs, while the ERN/Ne of HCs was associated with an additional activation in the posterior cingulate cortex (BAs 29 and 30) (Figure 5B). By contrast, the CRN map was associated with a main generator within medial frontal/dACC regions, equally in both groups. The maximum was localized within the superior frontal gyrus (BA6; MNI coordinates: 5x, -0y, +70z) (see Table 3).

Regarding the Pe component, sLORETA showed that its brain generators primarily involved a cluster encompassing different cingulate areas, namely BAs 23, 24 and 31 (see Figure 5C) and the insula (BA 13). This network was not evidenced for the posterior positivity map associated with hits during the same time interval. A direct comparison between the two groups revealed a significantly stronger recruitment of deep/ventral 
cingulate areas (BAs 23, 31 and 32; see Figure 5D) for HCs compared to MDD patients during the processing of errors, while MDD patients recruited superior frontal areas (BA6) during this later time interval (see Table 3).

\section{Discussion}

\section{Balanced behavioral accuracy between MDD patients and HCs}

On average MDD patients and HCs committed 25 response errors. A balanced accuracy between the two groups was an important requirement as the ERN varies in amplitude depending on the number of errors (and by extension, the number of error trials eventually included in the ERP averages; the fewer the error number, the larger the ERN component, see Gehring et al., 1993). An asymmetric accuracy between the two groups would potentially be problematic as any group difference (at the level of the ERN or Pe component) could then easily be explained by this factor alone. However, a balanced accuracy between the two groups cannot be taken as evidence that MDD is not associated with error-monitoring deficits at the behavioral level. This balanced accuracy could be explained by the use of a speeded Go/noGo task and an individually calibrated response deadline in the present case, cancelling out potential group differences. In the present study, this was clearly not the case. Although MDD patients were overall slower than HCs, the smaller Pe component for MDD patients compared to HCs was not explained by group differences in accuracy, or by the RT speed difference between groups (see complementary covariate analysis).

\section{Enhanced ERN/Ne in MDD}

The ERN/Ne results point to error monitoring impairments starting as early as $50 \mathrm{~ms}$ following error commission in MDD. The ERN/Ne was larger in size at the scalp level in MDD patients than in HCs when controlling for RT differences between the two groups. In this study, severely depressed individuals were included in the MDD group and this severity may potentially account for the lack of a larger ERN/Ne component. Previous ERP studies 
already reported similar or even diminished ERN/Ne amplitudes in severely depressed individuals who are characterized by apathy, anhedonia and psychomotor retardation (Schrijvers, de Bruijn, et al., 2008; Schrijvers et al., 2009; Schrijvers, Hulstijn, \& Sabbe, 2008).

The complementary topographical and source localization analyses confirmed that this early error monitoring process was qualitatively different at the neural level for MDD patients compared to HCs. The ERN/Ne component of MDD patients (as well as HCs) was related to increased activity in brain regions located primarily within the medial frontal gyrus (BA6) and dACC (BA24), as well as in the medial frontal gyrus (BA6) and in nonoverlapping posterior parietal regions (BA7, with an activation extending towards BA 31) (see Fig. 5A; see also Aarts \& Pourtois, 2010). The contribution of Premotor/Supplementary motor area and/or the dACC in early error monitoring processes (ERN component) is consistent with previous ERP and fMRI studies (Dehaene et al., 1994; Herrmann et al., 2004; O'Connell et al., 2007; Ullsperger \& von Cramon, 2004). Interestingly, the ERN/Ne of MDD patients was explained by an enhanced MFC/dLPFC (BA8 and BA9) activity, relative to HCs. A direct comparison between the two groups confirmed that MDD recruited extra dLPFC areas, during the time-course of the ERN component (i.e., BA6, BA8 and BA9; see Silton et al., 2011), that have previously been implicated in cognitive control processes (Botvinick, Braver, Barch, Carter, \& Cohen, 2001; Duncan \& Owen, 2000; Ridderinkhof, Ullsperger, Crone, \& Nieuwenhuis, 2004). Other studies (Hoehn-Saric, Lee, McLeod, \& Wong, 2005; Sinha, Mohlman, \& Gorman, 2004) have also related increased dlPFC activity to augmented ruminative thinking or worry, which is a hallmark of MDD (Nolen-Hoeksema, 2000). Accordingly, the observed enhanced dIPFC activity found in MDD patients during the early monitoring/detection of response errors (besides the normal dACC activation, shared 
with $\mathrm{HCs}$ ) might be related to ruminative processes, that would modulate the interplay between dIPFC and ACC during early stages of error-monitoring (see Pizzagalli, 2011).

Interestingly, these source localization results also corroborate previous imaging studies reporting hyperactive $\mathrm{dLPFC}$ in depressed patients during tasks involving conflict detection and resolution, including flanker or Stroop tasks (Wagner et al., 2006). A hyperactive dLPFC during conflict or error monitoring in MDD might reflect a compensatory mechanism meant to adjust for the deficient cognitive efficiency (Pizzagalli, 2011). Such a mechanism might eventually explain why the accuracy of MDD patients and HCs during the Go/noGo task was actually balanced in the present case.

\section{MDD is associated with a reduced Pe component}

Besides the ERN, the present results show that MDD patients have a substantially smaller Pe component than HCs during early error monitoring. This decreased Pe component during error monitoring in MDD patients might be explained either by symptom severity, which is stronger in MDD patients (the present study; Olvet, Klein, \& Hajcak, 2010; Schrijvers, de Bruijn et al., 2008; Schrijvers et al., 2009) than in moderately depressed individuals (Chiu \& Deldin, 2007; Compton et al., 2008; Holmes \& Pizzagalli, 2008).

Given the impaired motivation in MDD patients (DSM-IV-TR, APA, 2000), and the link between the Pe component and the motivational significance of an error (Overbeek et al., 2005), a reduced Pe component may be explained in terms of a change in the detection of an otherwise salient or behaviorally relevant event (i.e., unwanted response error). However, the post-error adjustment following errors (Rabbitt, 1966) and the total number of errors was comparable in MDD patients relative to HCs. This suggests that MDD patients were equally able to comply with the task demands compared to HCs and that a mere change in levels of "intrinsic" motivation during the task across the two groups did probably not account for the present ERP results. 
Finally, a blunted Pe component in MDD patients could stem from an exaggerated ruminative thinking style. In this view, the accumulation of evidence leading to the conscious detection of a response error, as reflected by the Pe component (Steinhauser \& Yeung, 2010), would be impaired since other intrusive thoughts may prevent its normal unfolding. This limited resource account is also consistent with the idea that the Pe reflects a "bottom-up" attentional orienting process, similar to the P300 component (Ridderinkhof et al., 2009). Presumably, if less "bottom-up" attention is allocated to the monitoring of actions and errors (because attention resources are used by a concurrent mental process, for example rumination), the monitoring and the conscious registration of these errors are probably less effective. Interestingly, previous studies already reported a decreased noGo P300 in depressed individuals (Ruchsow, Groen, \& Kiefer, 2008).

\section{MDD influences both stages of early error detection through modulations in different}

\section{brain networks}

More generally, the current ERP results, which are consistent with earlier findings obtained with comparable clinical samples (Schrijvers et al., 2009), suggest that early stages of error detection are different between MDDs and $\mathrm{HCs}$ at multiple levels through modulations in non-overlapping medial frontal and ACC networks. We did not find evidence for a differential effect of MDD at the level of the Pe, using standard scalp measurements. However, the complementing topographical and source localization results showed that these two consecutive stages of early error detection (ERN and Pe) were different in MDD patients compared to $\mathrm{HCs}$, due to the reliable modulation of specific and different brain networks:

while the ACC was overactive and additional dIPFC sources underlying the ERN were identified in MDD, the "normal" ventral ACC sources giving rise to the Pe component were substantially reduced in MDD. 
Presumably, these effects might reflect an inability or deficiency to treat or regulate the emotional value of actions early on following response onset, at the level of the ERN (Aarts, De Houwer, \& Pourtois, 2012; Aarts, De Houwer, \& Pourtois, 2013). Such an early deficient process could stem from abnormal prefrontal-based executive functions or alternatively an exaggerated ruminative thinking style, which might in turn consume resources used otherwise to process later the motivational significance or salience of response errors (Pe effect). Future studies are needed to establish whether rumination (or another process) might account for these abnormal early error monitoring processes seen in MDD.

\section{Limitations}

A few limitations should be noted. First, we could recruit 20 MDD patients and 20 HCs, which corresponds to a modest sample size. Although 20 participants per group is common in ERP research, increasing the sample size might have further increased the power. On the other hand, the complementary topographical and source localization results clearly showed that the present study was not underpowered, as we were able to reveal significant modulatory effects of MDD at two different moments following response error onset in nonoverlapping medial frontal and ACC regions.

Second, regular anti-depressant drugs may have either amplified or obscured some of the group differences found during error processing. However, these drugs have not yet been linked to systematic alterations of the amplitude or morphology of error-related ERP components in previous ERP studies using HCs (De Bruijn, Sabbe, Hulstijn, Ruigt, \& Verkes, 2006; Stern et al., 2010). Nonetheless, additional ERP studies are needed in order to assess whether systematic changes in early error monitoring brain processes seen in MDD patients (e.g., blunted Pe component) are modified by anti-depressant medication. 
To conclude, the present study reveals that MDD is associated with altered early error monitoring processes at multiple levels (ERN and Pe components) through impairments in different MFC and dLPFC brain networks. 


\section{Acknowledgments}

This work is supported by grants from the European Research Council (Starting Grant \#200758) and Ghent University (BOF Grant \#05Z01708). MAV is a postdoctoral fellow of the Research Foundation Flanders (FWO) (FWO08/PDO/168). We thank Monica Dhar for her feedback and suggestions on this manuscript. 


\section{References}

Aarts, K., De Houwer, J., \& Pourtois, G. (2012). Evidence for the automatic evaluation of self-generated actions. Cognition, 124(2), 117-127. doi: 10.1016/j.cognition.2012.05.009

Aarts, K., De Houwer, J., \& Pourtois, G. (2013). Erroneous and correct actions have a different affective value: Evidence from ERPs. Emotion.

Aarts, K., \& Pourtois, G. (2010). Anxiety not only increases, but also alters early errormonitoring functions. Cognitive, Affective, \& Behavioral Neuroscience, 10(4), 479-492. doi: 10.3758/CABN.10.4.479

American Psychiatric Association. (2000). Diagnostic and statistical manual of mental disorders (text revision). Washington, D.C: Author.

Beck, A. T., Steer, R. A., \& Brown, G. K. (1996). Manual for the Beck Depression Inventory-II. San Antonio, TX: Psychological Corporation.

Botvinick, M. M., Braver, T. S., Barch, D. M., Carter, C. S., \& Cohen, J. D. (2001). Conflict monitoring and cognitive control. Psychological Review, 108(3), 624-652. doi: 10.1037//0033-295X.108.3.624

Bush, G., Luu, P., \& Posner, M. I. (2000). Cognitive and emotional influences in anterior cingulate cortex. Trends in Cognitive Sciences, 4(6), 215-222. doi: 10.1016/S13646613(00)01483-2

Chiu, P. H., \& Deldin, P. J. (2007). Neural evidence for enhanced error detection in major depressive disorder. The American Journal of Psychiatry, 164(4), 608-616. doi: 10.1176/appi.ajp.164.4.608

De Bruijn, E. R. A., Sabbe, B. G. C., Hulstijn, W., Ruigt, G. S. F., \& Verkes, R. J. (2006). Effects of antipsychotic and antidepressant drugs on action monitoring in healthy volunteers. Brain Research, 1105, 122-129. doi: 10.1016/j.brainres.2006.01.006 
Debener, S., Ullsperger, M., Fiehler, K., von Cramon, D. Y., \& Engel, A. K. (2005). Monitoring error processing by means of simultaneous EEG/fMRI recordings II: Single-trial independent component analysis of the error-related negativity (ERN). Journal of Psychophysiology, 19(2), 111-111.

Dehaene, S., Posner, M. I., \& Tucker, D. M. (1994). Localization of a neural system for error-detection and compensation. Psychological Science, 5(5), 303-305. doi: 10.1111/j.14679280.1994.tb00630.x

Dhar, M., \& Pourtois, G. (2011). Early error detection is generic, but subsequent adaption to errors is not: Evidence from ERPs. Neuropsychologia, 49(5), 1236-1245. doi: 10.1016/j.neuropsychologia.2011.01.006

Duncan, J., \& Owen, A. M. (2000). Common regions of the human frontal lobe recruited by diverse cognitive demands. Trends in Neurosciences, 23(10), 475-483. doi: $10.1016 / \mathrm{S} 0166-2236(00) 01633-7$

Falkenstein, M., Hohnsbein, J., Hoormann, J., \& Blanke, L. (1991). Effects of crossmodal divided attention on late ERP components. II. Error processing in choice reaction tasks. Electroencephalography and Clinical Neurophysiology, 78(6), 447-455.

Falkenstein, M., Hoormann, J., Christ, S., \& Hohnsbein, J. (2000). ERP components on reaction errors and their functional significance: A tutorial. Biological Psychology, 51(2-3), 87-107. doi: 10.1016/S0301-0511(99)00031-9

Fuchs, M., Kastner, J., Wagner, M., Hawes, S., \& Ebersole, J. S. (2002). A standardized boundary element method volume conductor model. Clinical Neurophysiology, 113(5), 702712. doi: 10.1016/S1388-2457(02)00030-5

Gehring, W. J., Goss, B., Coles, M. G. H., Meyer, D. E., \& Donchin, E. (1993). A neural system for error-detection and compensation. Psychological Science, 4(6), 385-390. doi: 10.1111/j.1467-9280.1993.tb00586.x 
Gratton, G., Coles, M. G. H., \& Donchin, E. (1983). A new method for off-line removal of ocular artifact. Electroencephalography and Clinical Neurophysiology, 55(4), 468-484. doi: $10.1016 / 0013-4694(83) 90135-9$

Gruendler, T. O., Ullsperger, M., \& Huster, R. J. (2011). Event-related potential correlates of performance-monitoring in a lateralized time-estimation task. PLOS ONE, 6(10), e25591. doi: 10.1371/journal.pone.0025591

Hamilton, M. (1960). A rating scale for depression. Journal of Neurology, Neurosurgery and Psychiatry, 23, 56-62.

Herrmann, M. J., Rommler, J., Ehlis, A. C., Heidrich, A., \& Fallgatter, A. J. (2004). Source localization (LORETA) of the error-related negativity (ERN/Ne) and positivity (Pe). Cognitive Brain Research, 20(2), 294-299. doi: 10.1016/j.cogbrainres.2004.02.013

Hoehn-Saric, R., Lee, J. S., McLeod, D. R., \& Wong, D. F. (2005). Effect of worry on regional cerebral blood flow in nonanxious subjects. Psychiatry Research: Neuroimaging, 140, 259-269. doi: 10.1016/j.pscychresns.2005.05.013

Holmes, A. J., \& Pizzagalli, D. A. (2008). Spatiotemporal dynamics of error processing dysfunctions in major depressive disorder. Archives of General Psychiatry, 65(2), 179-188. doi: 10.1001/archgenpsychiatry.2007.19

Holmes, A. J., \& Pizzagalli, D. A. (2010). Effects of task-relevant incentives on the electrophysiological correlates of error processing in major depressive disorder. Cognitive, Affective \& Behavioral Neuroscience, 10(1), 119-128. doi: 10.3158/CABN.10.1.119

Jurcak, V., Tsuzuki, D., \& Dan, I. (2007). 10/20, 10/10, and 10/5 systems revisited: Their validity as relative head-surface-based positioning systems. Neuroimage, 34(4), 1600-1611. doi: 10.1016/j.neuroimage.2006.09.024

Laming, D. (1979). Autocorrelation of choice-reaction times. Acta Psychologica, 43(5), 381-412. doi: 10.1016/0001-6918(79)90032-5 
Lehmann, D., \& Skrandies, W. (1980). Reference-free identification of components of checkerboard-evoked multichannel potential fields. Electroencephalography and Clinical Neurophysiology, 48(6), 609-621. doi: 10.1016/0013-4694(80)90419-8

Mayberg, H. S. (1997). Limbic-cortical dysregulation: A proposed model of depression. Journal of Neuropsychology and Clinical Neuroscience, 9(3), 471-481.

Mazziotta, J., Toga, A., Evans, A., Fox, P., Lancaster, J., Zilles, K., . . . Mazoyer, B. (2001). A probabilistic atlas and reference system for the human brain: International Consortium for Brain Mapping (ICBM). Philosophical Transactions of the Royal Society. Series B, Biological Sciences, 356(1412), 1293-1322.

Michel, C. M., Seeck, M., \& Landis, T. (1999). Spatiotemporal dynamics of human cognition. News in Physiological Sciences, 14, 206-214.

Mineka, S., Watson, D., \& Clark, L. A. (1998). Comorbidity of anxiety and unipolar mood disorders. Annual Review of Psychology, 49, 377-412. doi: 10.1146/annurev.psych.49.1.377

Murray, M. M., Brunet, D., \& Michel, C. M. (2008). Topographic ERP analyses: A stepby-step tutorial review. Brain Topography, 20(4), 249-264. doi: 10.1007/s10548-008-0054-5

Nieuwenhuis, S., Ridderinkhof, K. R., Blow, J., Band, G. P. H., \& Kok, A. (2001). Errorrelated brain potentials are differentially related to awareness of response errors: Evidence from an antisaccade task. Psychophysiology, 38(5), 752-760. doi: $10.1017 / \mathrm{S} 0048577201001111$

Nolen-Hoeksema, S. (2000). The role of rumination in depressive disorders and mixed anxiety/depressive symptoms. Journal of Abnormal Psychology, 109(3), 504-511. doi: 10.1037/0021-843X.109.3.504

O'Connell, R. G., Dockree, P. M., Bellgrove, M. A., Kelly, S. P., Hester, R., Garavan, H., . . Foxe, J. J. (2007). The role of cingulate cortex in the detection of errors with and without 
awareness: A high-density electrical mapping study. European Journal of Neuroscience, 25(8), 2571-2579. doi: 10.1111/j.1460-9568.2007.05477.x

Olvet, D. M., \& Hajcak, G. (2008). The error-related negativity (ERN) and psychopathology: Toward an endophenotype. Clinical Psychology Review, 28(8), 1343-1354. doi: 10.1016/j.cpr.2008.07.003

Olvet, D. M., Klein, D. N., \& Hajcak, G. (2010). Depression symptom severity and errorrelated brain activity. Psychiatry Research, 179, 30-37. doi: 10.1016/j.psychres.2010.06.008

Overbeek, T. J. M., Nieuwenhuis, S., \& Ridderinkhof, K. R. (2005). Dissociable components of error processing: On the functional significance of the Pe vis-à-vis the ERN/Ne. Journal of Psychophysiology, 19(4), 319-329. doi: 10.1027/0269-8803.19.4.319

Pascual-Marqui, R. D. (2002). Standardized low-resolution brain electromagnetic tomography (sLORETA): Technical details. Methods and Findings in Experimental and Clinical Pharmacology, 24D, 5-12.

Picton, T. W., Bentin, S., Berg, P., Donchin, E., Hillyard, S. A., Johnson, R., . . Taylor, M. J. (2000). Guidelines for using human event-related potentials to study cognition: Recording standards and publication criteria. Psychophysiology, 37(2), 127-152. doi: $10.1017 / \mathrm{S} 0048577200000305$

Pizzagalli, D. A. (2011). Frontocingulate dysfunction in depression: Toward biomarkers of treatment response. Neuropsychopharmacology, 36(1), 183-206. doi: 10.1038/npp.2010.166

Pourtois, G. (2011). Early error detection predicted by reduced pre-response control process: an ERP topographic mapping study. Brain Topography, 23(4), 403-422. doi: $10.1007 / \mathrm{s} 10548-010-0159-5$

Pourtois, G., Delplanque, S., Michel, C., \& Vuilleumier, P. (2008). Beyond conventional event-related brain potential (ERP): Exploring the time-course of visual emotion processing 
using topographic and principal component analyses. Brain Topography, 20(4), 265-277. doi: $10.1007 / \mathrm{s} 10548-008-0053-6$

Pourtois, G., Vocat, R., N'diaye, K., Spinelli, L., Seeck, M., \& Vuilleumier, P. (2010). Errors recruit both cognitive and emotional monitoring systems: Simultaneous intracranial recordings in the dorsal anterior cingulate gyrus and amygdala combined with fMRI. Neuropsychologia, 48(4), 1144-1159. doi: 10.1016/j.neuropsychologia.2009.12.020

Rabbitt, P. M. (1966). Errors and error correction in choice-response tasks. Journal of Experimental Psychology, 71(2), 264-272.

Ridderinkhof, K. R., Ramautar, J. R., \& Wijnen, J. G. (2009). To Pe or not to Pe: A P3like ERP component reflecting the processing of response errors. Psychophysiology, 46(3), 531-538. doi: 10.1111/j.1469-8986.2009.00790.x

Ridderinkhof, K. R., Ullsperger, M., Crone, E. A., \& Nieuwenhuis, S. (2004). The role of medial frontal cortex in cognitive control. Science, 306, 443-447. doi: 10.1126/science. 1100301

Ruchsow, M., Groen, G., \& Kiefer, M. (2008). Electrophysiological evidence for reduced inhibitory control in depressed patients in partial remission: A Go/Nogo study. International Journal of Psychophysiology, 68(3), 209-218. doi: 10.1016/j.ijpsycho.2008.01.010

Ruchsow, M., Hernberger, B., Beschoner, P., Gron, G., Spitzer, M., \& Kiefer, M. (2006). Error processing in major depressive disorder: Evidence from event-related potentials. Journal of Psychiatric Research, 40(1), 37-46. doi: 10.1016/j.jpsychires.2005.02.002

Ruchsow, M., Hernberger, B., Wiesend, C., Gron, G., Spitzer, M., \& Kiefer, M. (2004). The effect of erroneous responses on response monitoring in patients with major depressive disorder: A study with event-related potentials. Psychophysiology, 41(6), 833-840. doi: 10.1111/j.1469-8986.2004.00237.x 
Schrijvers, D., de Bruijn, E. R., Maas, Y., De Grave, C., Sabbe, B. G., \& Hulstijn, W. (2008). Action monitoring in major depressive disorder with psychomotor retardation. Cortex, 44(5), 569-579. doi: 10.1016/j.cortex.2007.08.014

Schrijvers, D., De Bruijn, E. R., Maas, Y. J., Vancoillie, P., Hulstijn, W., \& Sabbe, B. G. (2009). Action monitoring and depressive symptom reduction in major depressive disorder. International Journal of Psychophysiology, 71(3), 218-224. doi: 10.1016/j.ijpsycho.2008.09.005

Schrijvers, D., Hulstijn, W., \& Sabbe, B. G. C. (2008). Psychomotor symptoms in depression: a diagnostic, pathophysiological and therapeutic tool. Journal of Affective Disorders, 109, 1-20. doi: 10.1016/j.jad.2007.10.019

Seifert, S., von Cramon, D. Y., Imperati, D., Tittgemeyer, T., \& Ullsperger, M. (2011). Thalamocingulate interactions in performance monitoring. Journal of Neuroscience, 31(9), $3375-3383$.

Silton, R. L., Heller, W., Towers, D. N., Engels, A. S., Edgar, J. C., Spielberg, J. M., . . Miller, G. A. (2011). Depression and anxious apprehension distinguish frontocingulate cortical activity during top-down attentional control. Journal of Abnormal Psychology, 120(2), 272-285. doi: 10.1037/a0023204

Sinha, S., Mohlman, J., \& Gorman, J. M. (2004). Neurobiology of generalized anxiety disorder. In R. G. Heimberg, C. L. Turk \& D. S. Mennin (Eds.), Generalized Anxiety Disorder: Advances in Research and Practice (pp. 187-216). New York: Guilford.

Steinhauser, M., \& Yeung, N. (2010). Decision processes in human performance monitoring. Journal of Neuroscience, 30(46), 15643-15653. doi: 10.1523/JNEUROSCI.189910.2010

Stern, E. R., Liu, Y., Gehring, W. J., Lister, J. J., Yin, G., Zhang, J., . . Taylor, S. F. (2010). Chronic medication does not affect hyperactive error responses in obsessive- 
compulsive disorder. Psychophysiology, 47(5), 913-920. doi: 10.1111/j.14698986.2010.00988.x

Ullsperger, M., \& von Cramon, D. Y. (2004). Neuroimaging of performance monitoring: Error detection and beyond. Cortex, 40(4-5), 593-604. doi: 10.1016/S0010-9452(08)70155-2

Wagner, G., Sinsela, E., Sobanskic, T., Köhlera, S., Marinoua, T., Mentzelb, H., . . . Schlössera, R. G. M. (2006). Cortical inefficiency in patients with unipolar depression: An event-related fMRI study with the Stroop task. Biological Psychiatry, 59(10), 958-965. doi: 10.1016/j.biopsych.2005.10.025 
Table 1

Descriptive statistics for healthy controls $(H C)$ and depressed patients (MDD)

\begin{tabular}{|c|c|c|c|}
\hline & $\mathrm{HC}$ & MDD & \\
\hline & $M(S E M)$ & $M(S E M)$ & $p$ \\
\hline $\bar{N}$ & 20 & 20 & \\
\hline Age & $38.95(3.43)$ & $36.90(2.85)$ & .65 \\
\hline Sex & $3 \mathrm{M} / 17 \mathrm{~F}$ & $10 \mathrm{M} / 10 \mathrm{~F}$ & $<.05$ \\
\hline Education* & $2.06(.20)$ & $1.69(.22)$ & .22 \\
\hline HAM_D & $0.24(0.14)$ & $28.12(1.33)$ & $<.001$ \\
\hline BDI_II & $1.59(0.97)$ & $33.24(2.87)$ & $<.001$ \\
\hline MDD with comorbid anxiety & & $n=3$ & \\
\hline Treatment resistance $* *$ & & $n=9$ & \\
\hline Age at onset $(n=17)$ & & $30.76(3.20)$ & \\
\hline Length of episode (months; $n=17$ ) & & $7.35(1.38)$ & \\
\hline Number of episodes $(n=17)$ & & $2.76(.35)$ & \\
\hline
\end{tabular}

Note that for the age of onset, length of episode and number of episodes, the data reported are for 17 MDD patients. These data for 3 MDD patients could not be accessed and saved for confidentiality reasons.

*Education: $0=$ primary school; $1=3$ years of high school; $2=6$ years of high school and $3=$ higher education

** Treatment resistance $=$ had taken anti-depressant medication for at least 7 months prior to testing 
Table 2

Accuracy and RTs in the Go/noGo task, separately for healthy controls (HC) and depressed patients (MDD)

\begin{tabular}{|c|c|c|c|c|c|c|}
\hline & & \multicolumn{2}{|l|}{$\mathrm{HC}$} & \multicolumn{2}{|l|}{ MDD } & \multirow[b]{2}{*}{$P$} \\
\hline & & $M$ & SEM & $M$ & SEM & \\
\hline \multirow[t]{4}{*}{ Number } & Error & 29.00 & 4.43 & 21.71 & 3.18 & .19 \\
\hline & Hit & 232.76 & 3.65 & 223.18 & 5.56 & .16 \\
\hline & Post-error & 65.07 & 2.16 & 62.47 & 3.65 & .16 \\
\hline & Post-hit & 65.27 & 0.58 & 65.13 & 0.47 & .19 \\
\hline \multirow[t]{4}{*}{ Speed } & Error & 263.79 & 9.49 & 316.07 & 20.21 & .03 \\
\hline & Hit & 325.31 & 12.15 & 358.35 & 16.32 & .11 \\
\hline & Post-error & 345.95 & 12.80 & 363.67 & 18.73 & .44 \\
\hline & Post-hit & 321.86 & 12.74 & 356.53 & 16.45 & .11 \\
\hline
\end{tabular}


Table 3

MNI coordinates of the differential error-related peak activations between HCs and MDD patients, separately for the ERN/Ne and Pe component

\begin{tabular}{|c|c|c|c|c|c|c|c|}
\hline \multirow[t]{2}{*}{ Component } & \multirow[t]{2}{*}{ Regions of Interest (ROI) } & \multicolumn{5}{|c|}{ MNI Coordinates } & \multirow{2}{*}{$\begin{array}{l}\text { sLORETA } \\
\text { p-values }\end{array}$} \\
\hline & & $\mathrm{BA}$ & $\mathrm{x}$ & $\mathrm{y}$ & $\mathrm{Z}$ & & \\
\hline \multirow[t]{5}{*}{$\mathrm{ERN} / \mathrm{Ne}$} & Superior frontal gyrus & 6 & 5 & 0 & 70 & $\mathrm{MDD}>\mathrm{HC}$ & $* *$ \\
\hline & & 8 & -25 & 25 & 45 & $\mathrm{MDD}>\mathrm{HC}$ & $*$ \\
\hline & & 9 & -10 & 35 & 35 & $\mathrm{MDD}>\mathrm{HC}$ & $*$ \\
\hline & Posterior cingulate & 30 & 25 & -55 & 0 & $\mathrm{MDD}<\mathrm{HC}$ & $* * *$ \\
\hline & & 29 & 10 & -45 & 5 & $\mathrm{MDD}<\mathrm{HC}$ & $* * *$ \\
\hline \multirow[t]{5}{*}{$\mathrm{Pe}$} & Insula & 13 & 35 & -15 & 20 & $\mathrm{MDD}<\mathrm{HC}$ & $* * *$ \\
\hline & Cingulate gyrus & 23 & 0 & -55 & 15 & $\mathrm{MDD}<\mathrm{HC}$ & $* * *$ \\
\hline & & 31 & 5 & -60 & 20 & $\mathrm{MDD}<\mathrm{HC}$ & $* *$ \\
\hline & & 32 & -20 & 45 & 10 & $\mathrm{MDD}<\mathrm{HC}$ & $* * *$ \\
\hline & Superior frontal gyrus & 6 & 5 & 0 & 70 & $\mathrm{MDD}>\mathrm{HC}$ & $*$ \\
\hline
\end{tabular}

Note. $* p<.05 ; * * p<.01 ; * * * p<.001$ 


\section{Figure caption}

$\mathbf{A}$

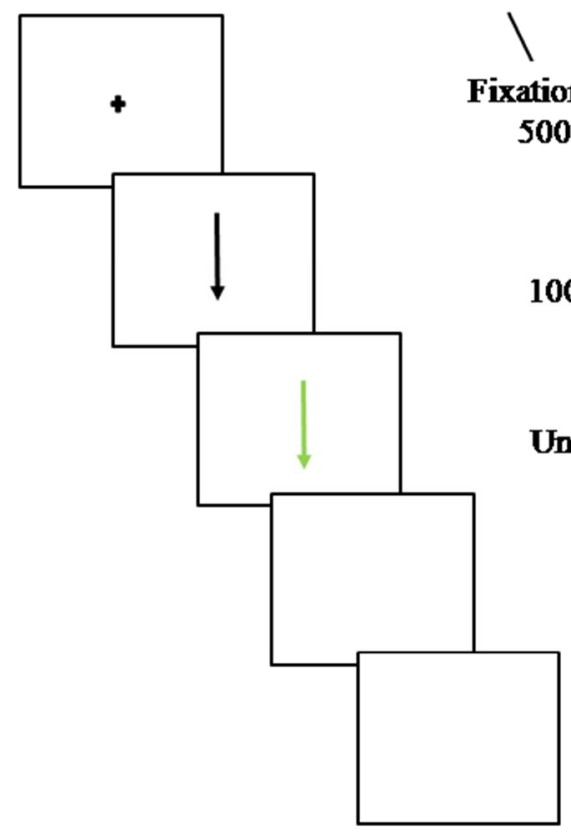

B

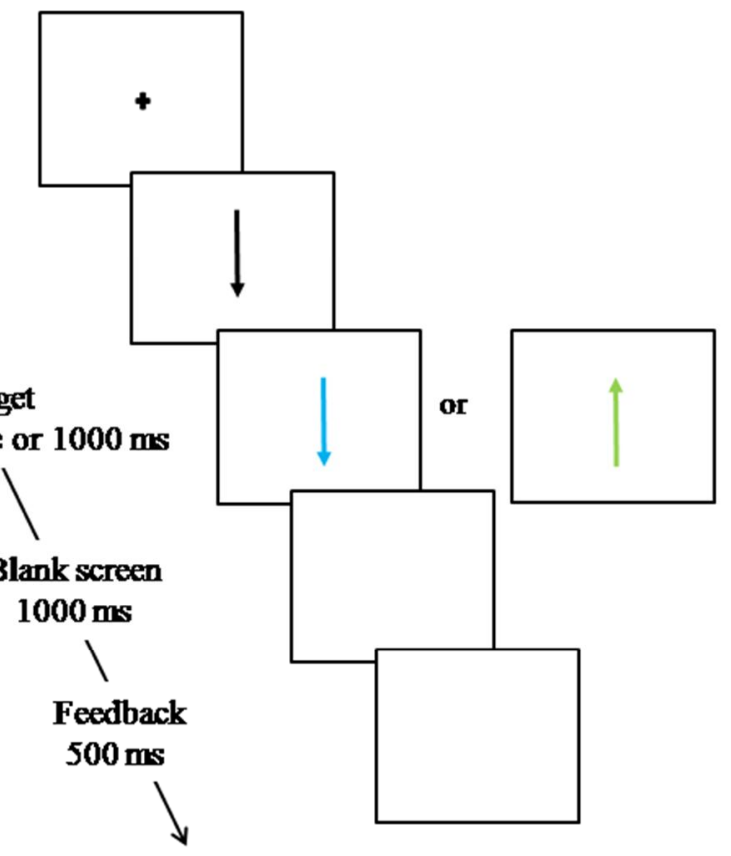

Figure 1. Stimuli and task. (A) On each trial, a black arrow was presented. After a variable interval (1000-2000 ms), the black arrow usually (2/3 $3^{\text {rds }}$ - Go trials) became green and kept its initial orientation (either up or down). (B) On the remaining $1 / 3^{\text {rd }}$ of the (noGo) trials, it became either turquoise and/or green but with a change in orientation (noGo trials). 
$\mathbf{A}$

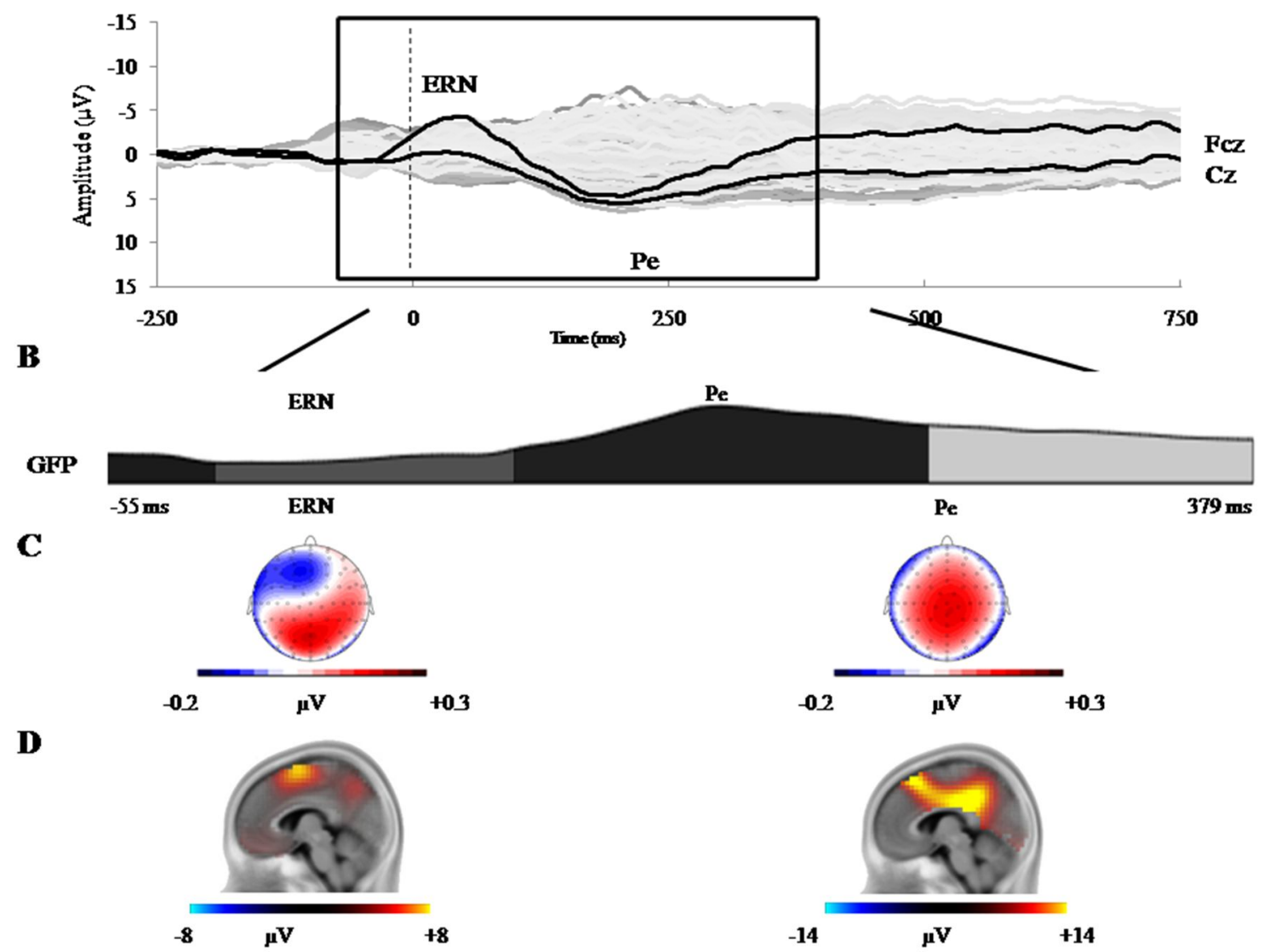

Figure 2. Identification of the ERN/Ne and Pe topographical components, and their intracerebral generators. (A) 128 electrodes were used and time-frames spanning from $-55 \mathrm{~ms}$ to $379 \mathrm{~ms}$ after response onset, encompassing the ERN and Pe. A butterfly view of the grandaverage ERP data of HCs (errors) from -500 to $+1000 \mathrm{~ms}$ around the response is shown (with two representative electrodes, FCZ and $\mathrm{Cz}$ shown in black), as well as the corresponding time interval selected for the segmentation in topographical components. (B) Two topographical maps, the ERN/Ne and Pe, were isolated using a clustering method. (C) These two maps corresponded to the ERN/Ne and Pe component. (D) SLORETA was used to gain insight into the neural generators underlying these dominant scalp configurations. For HCs, the MFC/dACC was estimated as the main neural generator of the ERN, while non-overlapping cingulate areas and the insula were found to account for the Pe component. 
$\mathbf{A}$

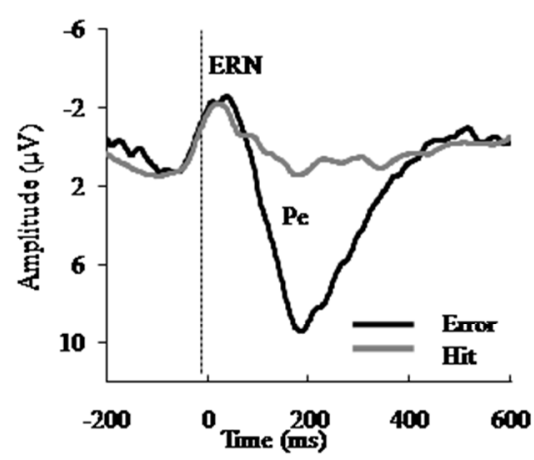

C

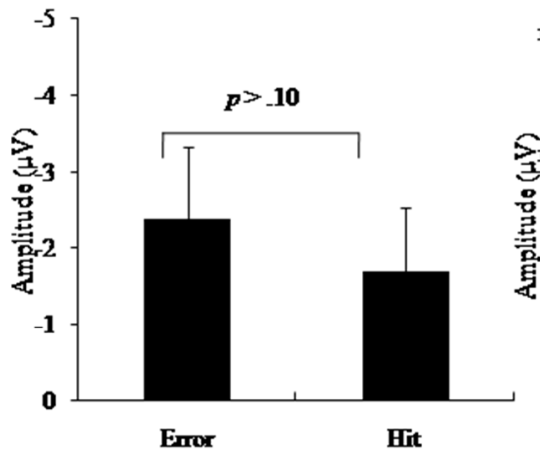

$\mathbf{E}$

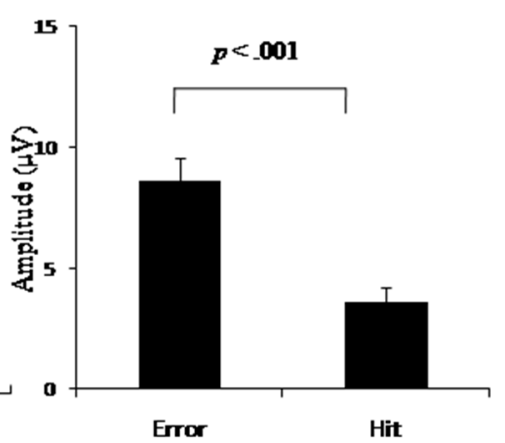

$\mathbf{B}$

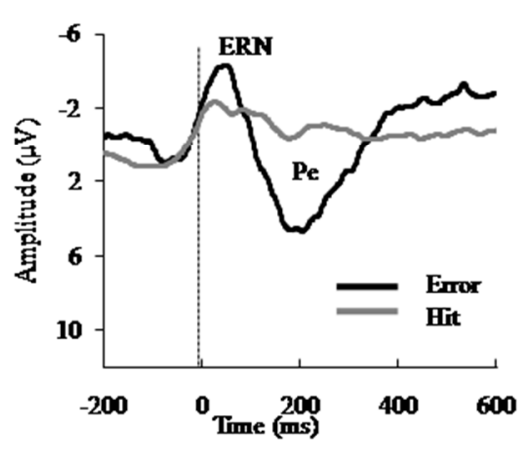

D

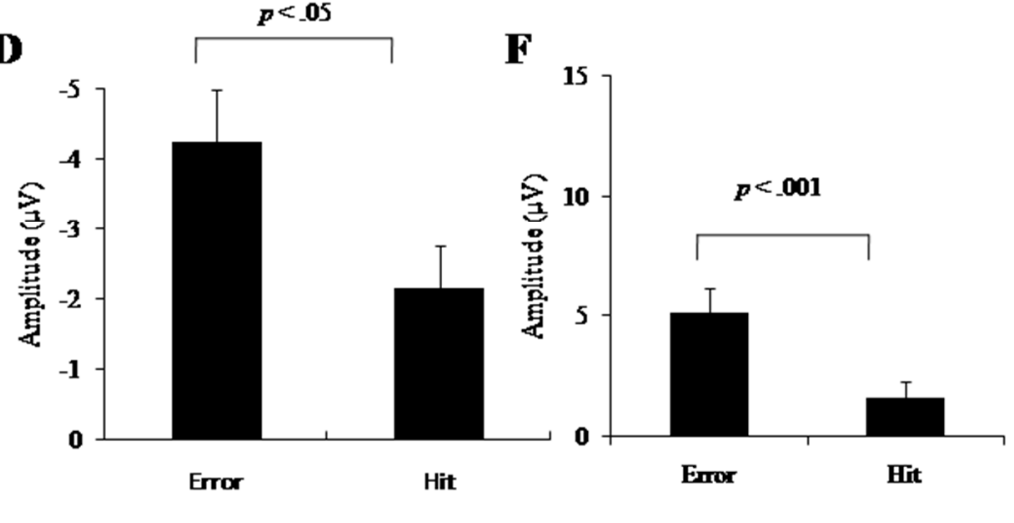

Figure 3. (A) Grand average ERP waveforms (electrode $\mathrm{FCz}$ ) for the $\mathrm{HCs}$ and (B) for the MDD patients. (C) Mean amplitude $(\mu \mathrm{V}) \pm 1$ standard error of the mean (S.E.M) of the ERN/Ne for errors vs. hits in HCs and (D) in MDD patients. (E) Mean amplitude $(\mu \mathrm{V}) \pm 1$ S.E.M of the Pe for errors vs. hits in HCs and (F) in MDD patients. 
$\mathbf{A}$

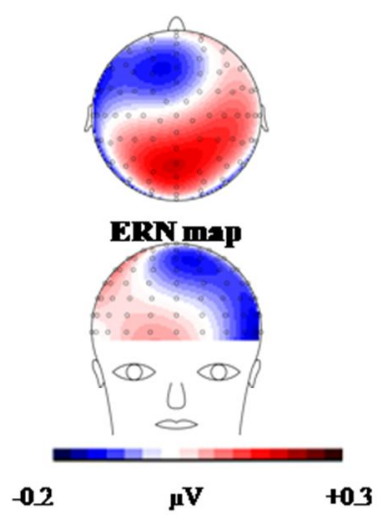

$\mathbf{C}$

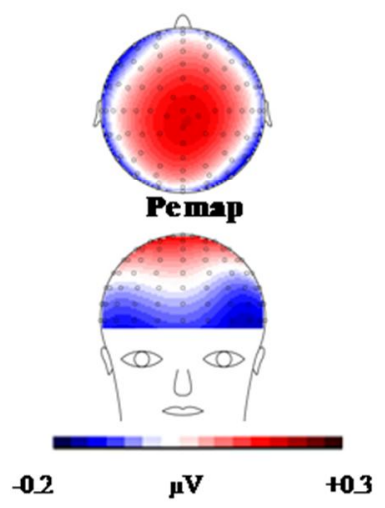

B
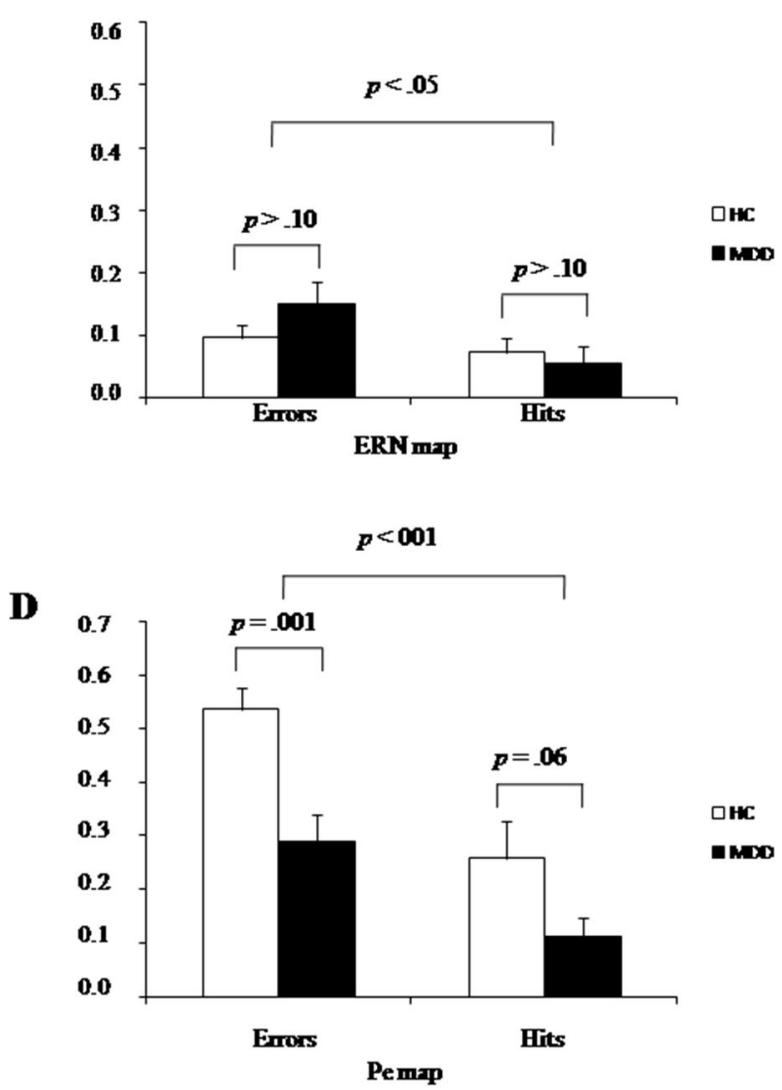

Figure 4. Topographical components during the time interval (-10 to $90 \mathrm{~ms}$ around response onset) corresponding either to the ERN/Ne or Pe. (A) The scalp map of the ERN/Ne showed a negative activity reaching its maximum amplitude at $\mathrm{FCz}$ electrode position, and extending towards left lateral frontal electrodes. (B) The ERN/Ne topographical component explained more variance for errors than for hits, without a clear modulation by MDD. (C) The scalp map of the Pe was characterized by a broad positive activity over central electrode positions. (D) The Pe topographical component explained more variance for errors than for hits, especially for HCs compared to MDD patients. 

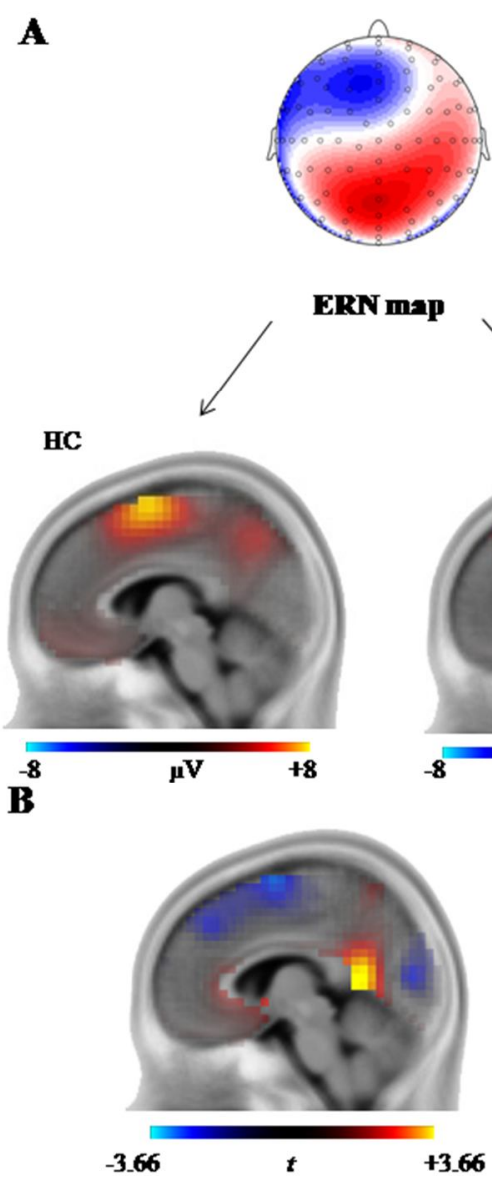

C
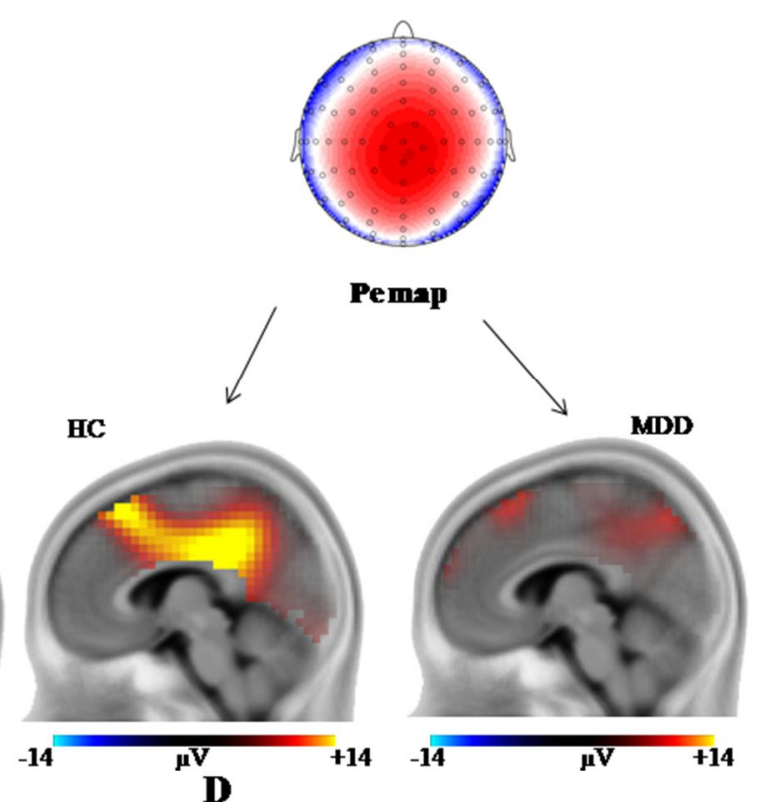

D
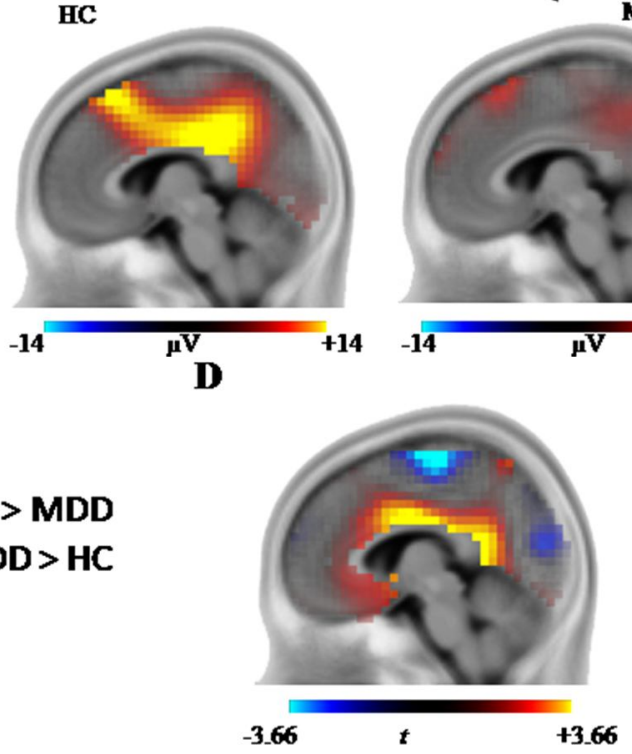

Figure 5. Source localization results (sLORETA). (A) Inverse solution for the ERN/Ne, separately for HCs and MDD patients, revealing a main cluster in the dACC (BAs 32 and 24) and MFC (superior frontal gyrus - BA 6). (B) MDD patients had a stronger MFC/dLPFC (BAs 6, 8 and 9) activation compared to $\mathrm{HCs}$, while the ERN/Ne of HCs was associated with an additional activation in the posterior cingulate cortex (BAs 29 and 30) (C) Inverse solution for the Pe, separately for HCs and MDD patients, revealing a main cluster in the cingulate areas (BAs 23, 24 and 31) in the former participants, but not the patients. (D) HCs recruited more ventral cingulate areas (BAs 23, 31 and 32) as well as insula regions (BA13, not shown on this view) than MDD patients. By contrast, MDD patients recruited additional superior frontal areas (BA6). 